\title{
A MyD88-dependent IFN $\gamma R-C C R 2$ signaling circuit is required for mobilization of monocytes and host defense against systemic bacterial challenge
}

\author{
Eric M Pietras ${ }^{1}$, Lloyd S Miller ${ }^{1,2}$, Carl T Johnson ${ }^{1}$, Ryan M O’Connell ${ }^{1}$, Paul W Dempsey ${ }^{1,5}$, Genhong Cheng ${ }^{1,2,3,4}$ \\ ${ }^{I}$ Department of Microbiology, Immunology and Molecular Genetics, David Geffen School of Medicine; ${ }^{2}$ Division of Dermatology, \\ Department of Medicine, David Geffen School of Medicine; ${ }^{3}$ Molecular Biology Institute, University of California, Los Angeles, \\ Los Angeles, CA 90095, USA; ${ }^{4}$ Jonsson Comprehensive Cancer Center, University of California, Los Angeles, Los Angeles, CA \\ 90095, USA
}

Monocytes are mobilized to sites of infection via interaction between the chemokine MCP-1 and its receptor, CCR2, at which point they differentiate into macrophages that mediate potent antimicrobial effects. In this study, we investigated the mechanisms by which monocytes are mobilized in response to systemic challenge with the intracellular bacterium Francisella tularensis. We found that mice deficient in MyD88, interferon- $\gamma$ (IFN $\gamma$ )R or CCR2 all had defects in the expansion of splenic monocyte populations upon $F$. tularensis challenge, and in control of $F$. tularensis infection. Interestingly, MyD88-deficient mice were defective in production of IFN $\gamma$, and IFN $\gamma R$ deficient mice exhibited defective production of MCP-1, the ligand for CCR2. Transplantation of IFN $\gamma$ R-deficient bone marrow (BM) into wild-type mice further suggested that mobilization of monocytes in response to $F$. tularensis challenge required IFN $\gamma \mathrm{R}$ expression on BM-derived cells. These studies define a critical host defense circuit wherein MyD88-dependent IFN $\gamma$ production signals via IFN $\gamma \mathrm{R}$ expressed on BM-derived cells, resulting in MCP-1 production and activation of CCR2-dependent mobilization of monocytes in the innate immune response to systemic $F$. tularensis challenge.

Keywords: bacteria; interferon; chemokine; monocyte; infection

Cell Research (2011) 21:1068-1079. doi:10.1038/cr.2011.59; published online 5 April 2011

\section{Introduction}

The mammalian organism is confronted at all times with a vast microbial menagerie consisting of a range of bacterial species, some of which are pathogenic and must be eliminated from the host. The mammalian immune system has developed a specialized set of immune mechanisms to detect and combat these pathogenic microorganisms [1]. The initial immune response is mediated by innate immunity, which is characterized by the rapid induction of an inflammatory response designed

Correspondence: Genhong Cheng

Tel: +310-825-8896; Fax: +310-206-5553

E-mail: gcheng@mednet.ucla.edu

${ }^{5}$ Current address: Cynvenio Biosystems LLC, Westlake Village, CA 91361, USA

Received 9 August 2010; revised 25 October 2010; accepted 15 December 2010; published online 5 April 2011 to control infection and activate the subsequent adaptive response. The innate immune response is induced via the recognition of pathogen-associated molecular patterns by an array of pattern recognition receptors such as the Tolllike receptors (TLRs) and NOD-like receptors (NLRs) $[2,3]$. These in turn lead to the activation of an array of cytokines, chemokines, adhesion molecules and antimicrobial peptides that result in proinflammatory immune responses that promote elimination of the pathogen $[2,4]$.

Upon pathogen recognition, a defining feature of the innate immune response is the mobilization and trafficking of myeloid cells, including neutrophils and monocytes, to infected tissues, where they phagocytose and kill pathogenic microbes [5]. In particular, monocytes have been shown to play a critical role in the control and elimination of intracellular bacteria such as Listeria monocytogenes, which has been extensively used as a model pathogen for studying the innate immune response 
to intracellular bacterial infection [6]. Mice deficient in CCR2, the receptor for the monocyte chemokines MCP1 and MCP-3, fail to mobilize monocyte populations in response to infection by intracellular bacteria and have impaired bacterial clearance [7-9]. In response to $L$. monocytogenes infection, Ly6C + monocytes accumulate in the spleen, where they mature into TNF- $\alpha$ - and iNOSproducing inflammatory monocytes [10]. These cells in turn act as antimicrobial effector cells and can promote the generation of adaptive T-cell responses [10].

Activation of effective monocyte responses to intracellular bacteria such as $F$. tularensis is enhanced via production of interferon- $\gamma$ (IFN $\gamma$ ) by activated NK and NKT cells early on, and subsequently by antigen-specific activation of CD4+ Th1 cell subsets [11-13]. IFN $\gamma$ has been shown to induce antimicrobial genes such as iNOS and LRG47, as well as to promote nutrient sequestration and the expression of antigen presentation molecules in multiple cell types $[14,15]$. Although the role of IFN $\gamma$ in the activation of antimicrobial genes in monocytes is well characterized, a potential role of IFN $\gamma$ in regulating other innate immune system functions, particularly the mobilization of myeloid cells, is not well defined.

In the present study, the mechanism by which monocytes are mobilized to sites of infection, in particular the spleen, after systemic Francisella tularensis challenge was investigated. F. tularensis is a gram-negative, intracellular bacterium that is the causative agent of tularemia, a potentially fatal zoonosis in humans if inoculation occurs via the pulmonary route [16]. Recognition of $F$. tularensis by the innate immune system is largely mediated by TLR2 and MyD88 [16, 17] and requires myeloid cells, including monocytes, as depletion of myeloid cells in mice using an anti-Gr-1 antibody (which recognizes the monocyte surface marker Ly6C as well as the granulocyte marker Ly6G) results in uncontrolled infection [18]. Further, resolution of $F$. tularensis infection requires IFN $\gamma$ signaling and the activation of antimicrobial genes including TNF- $\alpha$ and iNOS [19-22].

Although prior work has suggested that IFN $\gamma$ production plays a role in the formation of hepatic granulomas in mice upon $F$. tularensis challenge, the mechanism by which IFN $\gamma$ mediates myeloid cell trafficking, and the identity and the functional importance of the mobilized cells have not been established [23]. In the present study, we demonstrate that IFN $\gamma \mathrm{R}$ signaling in bone marrow (BM)-derived cells is required for the production of the monocyte chemokine MCP-1 and mobilization of monocyte populations in the spleen following bacterial challenge. Furthermore, we show the production of IFN $\gamma$ and resultant MCP-1 in response to systemic $F$. tularensis challenge occurs via an MyD88-dependent mechanism.
Lastly, effective control of $F$. tularensis infection requires TNF- $\alpha$ and NO production by the IFN $\gamma$ R/CCR2-mobilized monocytes. Taken together, this research identifies a new MyD88-dependent signaling mechanism proceeding via IFN $\gamma \mathrm{R} / \mathrm{CCR} 2$ that governs the mobilization and activation of monocytes in response to challenge with a systemic intracellular bacterium.

\section{Results}

IFN $R$ R expression is required for $M C P-1$ production and expansion of monocyte populations in the spleen after systemic $F$. tularensis challenge

IFN $\gamma \mathrm{R}$ signaling is a crucial mediator of immune responses to intracellular bacterial pathogens, including F. tularensis $[19,24]$. Thus, we asked whether IFN $\gamma \mathrm{R}$ signaling participates not only in the activation of antimicrobial mechanisms but also in the mobilization of monocytes during intracellular bacterial challenge using an established systemic $F$. tularensis infection model in mice [19, 25]. Wild-type (WT) and IFN $\gamma$ R-deficient mice were inoculated intravenously with $2 \times 10^{4}$ colony-forming units (CFUs) of $F$. tularensis, and, consistent with prior published results [19, 25], IFN $\gamma$ R-deficient mice but not WT mice were highly susceptible to this F. tularensis challenge (Supplementary information, Figure S1). These data indicate that IFN $\gamma \mathrm{R}$ signaling is critical for host defense against $F$. tularensis. To assess how IFN $\gamma \mathrm{R}$ signaling affects monocyte populations in the spleen after infection, we harvested spleens from infected WT and IFN $\gamma$ R-deficient mice and from uninfected control mice $24 \mathrm{~h}$ after $F$. tularensis challenge (Figure 1A-1C). We observed a significant expansion of $\mathrm{CD} 11 \mathrm{~b}+\mathrm{Ly} 6 \mathrm{C}^{\mathrm{hi}}$ monocytes in the spleens of WT mice $24 \mathrm{~h}$ after infection. In contrast, there was no increase in splenic monocytes in IFN $\gamma$ R-deficient mice relative to uninfected controls. On the other hand, IFN $\alpha \beta R$-deficient mice did not exhibit susceptibility to infection or defective expansion of monocyte populations after inoculation with $F$. tularensis, indicating that type I interferons are not required for host defense against $F$. tularensis (Supplementary information, Figure S1 and data not shown). These findings suggest that IFN $\gamma \mathrm{R}$ signaling is crucial for the expansion of splenic monocyte populations after systemic F. tularensis challenge.

Previous work has described a critical role for the monocyte chemokine MCP-1 in mobilizing monocytes to the periphery in response to systemic bacterial challenge. As there was a defective expansion of monocyte populations in the spleens of IFN $\gamma$ R-deficient mice in response to systemic $F$. tularensis challenge (Figure 1A-1C), we hypothesized that this may be related to defective MCP- 

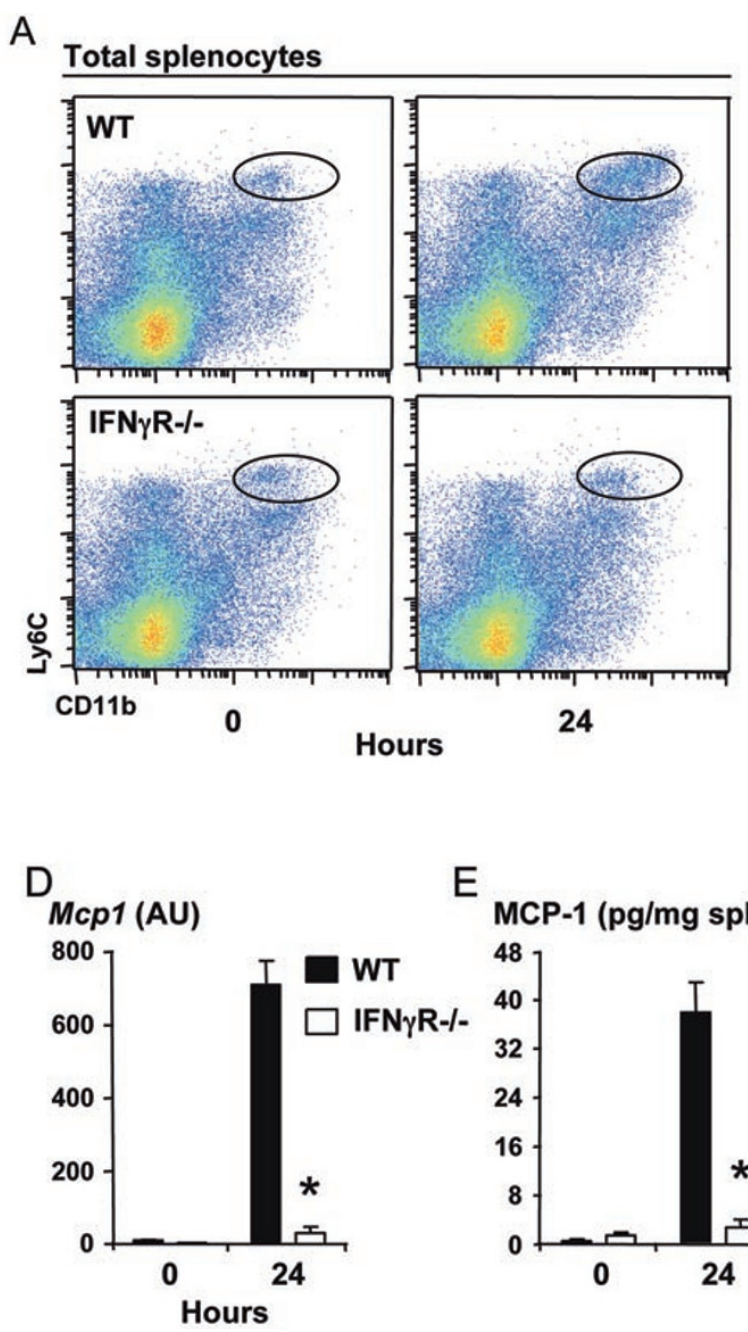

E
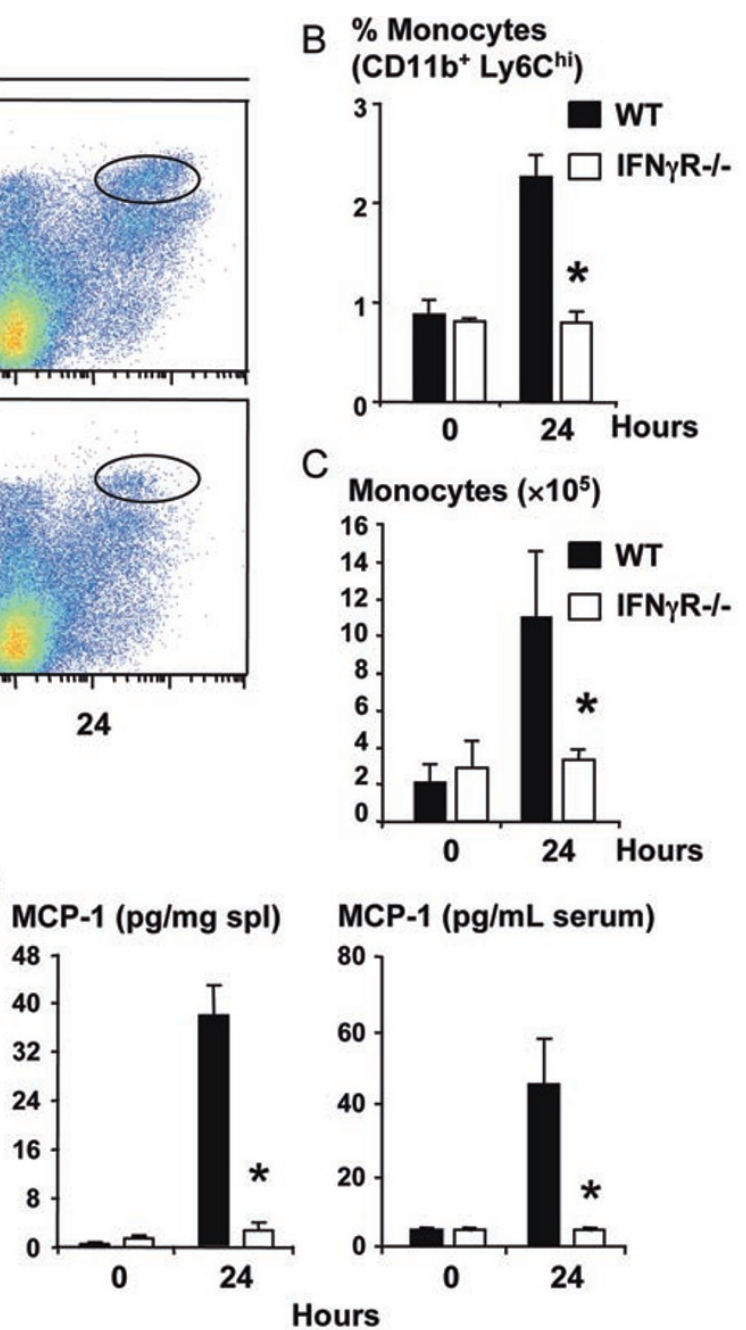

Figure 1 IFN $\gamma$ R expression is required for MCP-1 production and expansion of monocyte populations in the spleen after systemic F. tularensis challenge. WT and IFN $\gamma \mathrm{R}-/-$ mice $(n=3 / \mathrm{grp})$ were inoculated intravenously with $2 \times 10^{4} \mathrm{CFU}$ F. tularensis. After $24 \mathrm{~h}$, infected mice were euthanized along with uninfected $(0 \mathrm{~h})$ controls ( $n=3 / \mathrm{grp})$. (A) Representative analysis dot plot of RBC-depleted total splenocytes showing monocytes (CD11b+, Ly6C hi population) in control and F. tularensis-infected WT and IFN $\gamma \mathrm{R}-/-$ mice. Monocyte populations are shown as (B) mean proportion of total splenocytes \pm SD obtained from dot plots described in (A). (C) Mean absolute number of splenic monocytes \pm SD. (D) Mean mRNA levels \pm SD of MCP-1 from control and infected WT and IFN $\gamma$ R-/- mice. (E) Mean protein levels \pm SD of MCP-1 from spleen homogenates and sera of WT and IFN $\gamma$ R-deficient mice. * $p<0.05$ WT versus IFN $\gamma$ R-/- mice. Data are representative of at least five repeated experiments.

1 production in these mice. We therefore determined the induction of MCP-1 mRNA in the spleens of WT mice, IFN $\gamma R$-deficient mice, and uninfected control mice by Q-PCR $24 \mathrm{~h}$ after $F$. tularensis inoculation. There was a profound impairment in MCP-1 mRNA induction in the spleens of IFN $\gamma \mathrm{R}$-deficient mice compared with WT mice after $F$. tularensis infection (Figure 1D). Similarly, MCP-1 protein production as measured by ELISA was also severely impaired in the spleens and sera of IFN $\gamma \mathrm{R}-$ deficient mice compared with WT control mice after $F$. tularensis infection (Figure 1E). Collectively, these data reveal a crucial role for IFN $\gamma \mathrm{R}$ signaling in the activation of MCP-1 production and the subsequent expansion of splenic monocyte populations.

IFN $\gamma R$ expression in the BM compartment is necessary for MCP-1 production and expansion of monocyte populations in the spleen after systemic F. tularensis challenge

IFN $\gamma \mathrm{R}$ is expressed in a wide range of tissues, including BM-derived and non-BM-derived cell types. Therefore, we wanted to determine which compartment 
utilized IFN $\gamma$ R signaling to regulate expansion of splenic monocyte populations in response to systemic F. tularensis infection. WT recipient mice were lethally irradiated and reconstituted with either WT or IFN $\gamma$ R-deficient $\mathrm{BM}$, generating WT $\mathrm{BM} \rightarrow \mathrm{WT}$ and IFN $\gamma \mathrm{R}$-deficient $\mathrm{BM} \rightarrow$ WT chimeric mice. Eight weeks after reconstitution, these mice were inoculated intravenously with $F$. $t u$ larensis. WT $\mathrm{BM} \rightarrow \mathrm{WT}$ mice had increased proportions of monocytes in their spleens $24 \mathrm{~h}$ after infection; in contrast, IFN $\gamma \mathrm{R}$-deficient $\mathrm{BM} \rightarrow \mathrm{WT}$ mice had virtually no expansion of splenic monocytes (Figure 2A).

Further, a similar defect in splenic MCP-1 levels in $F$. tularensis-infected IFN $\gamma \mathrm{R}$-deficient $\mathrm{BM} \rightarrow \mathrm{WT}$ chimeric mice was observed compared with WT BM $\rightarrow$ WT control mice (Figure 2B). These data suggest that IFN $\gamma \mathrm{R}$ expression in BM-derived cells is required for MCP-1 production and the mobilization of monocyte populations in response to F. tularensis challenge. Similar to IFN $\gamma \mathrm{R}-$ deficient mice, IFN $\gamma \mathrm{R}$-deficient $\mathrm{BM} \rightarrow \mathrm{WT}$ chimeric mice had a substantially increased bacterial burden in the spleen, suggesting that $\mathrm{BM}$ expression of IFN $\gamma \mathrm{R}$ is also required for host defense against $F$. tularensis (Figure 2C).

IFN $\gamma R$ signaling induces $M C P-1$ production in BMderived cells and acts synergistically with innate sensing mechanisms to induce MCP-1 in vitro

Our data suggest that BM expression of IFN $\gamma \mathrm{R}$ is necessary for the production of MCP-1 in response to systemic F. tularensis challenge. MCP-1 can be produced by monocytes/macrophages, which arise from the BM [26-28]. Thus, we evaluated the ability of IFN $\gamma$ to induce $\mathrm{MCP}-1$ production, using primary $\mathrm{BM}$-derived macrophages (BMMs) as a model. Treatment of BMMs with IFN $\gamma$ resulted in significantly increased MCP-1 mRNA expression as determined by QPCR (Figure 3A). Furthermore, the effect of IFN $\gamma$ on MCP-1 protein production was dose-dependent, as determined by ELISA $24 \mathrm{~h}$ after stimulation (Figure 3B).

During systemic infection, monocytes and macrophages in the spleen likely encounter both $F$. tularensis and IFN $\gamma$ simultaneously. As pattern recognition receptors such as the TLRs also activate MCP-1 expression, we assessed whether IFN $\gamma \mathrm{R}$ and TLRs interact in the induction of MCP-1 expression. As F. tularensis can be recognized via TLR2 [16], BMMs were stimulated with the TLR2 ligand Pam3CysK in the presence or absence of IFN $\gamma$. Interestingly, a synergistic induction of MCP-1 mRNA was observed in BMMs treated with both Pam3CysK and IFN $\gamma$ (Figure 3C). Furthermore, to more closely simulate our in vivo bacterial challenge model, BMMs were cultured with $F$. tularensis in the presence or absence of IFN $\gamma$ and culture supernatants were harvested at $24 \mathrm{~h}$ for analysis of MCP-1 levels by ELISA. F. tularensis infection alone induced a modest amount of MCP-1 protein. However, addition of IFN $\gamma$ to the F. tularensis-stimulated $\mathrm{BMM}$ cultures induced a synergistic increase in MCP1 production, which was similar to our results with the
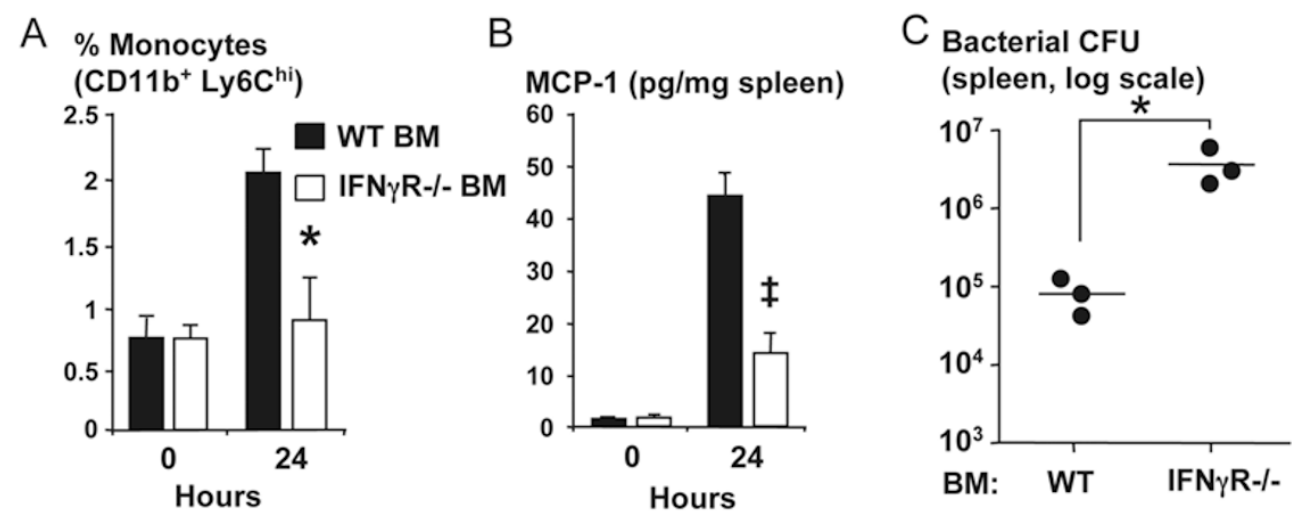

Figure 2 IFN $\gamma$ R expression in the bone marrow compartment is necessary for MCP-1 production and expansion of monocyte populations in the spleen after systemic F. tularensis challenge. WT BM $\rightarrow$ WT and IFN $\gamma \mathrm{R}-/-\mathrm{BM} \rightarrow \mathrm{WT}$ mice $(n=3 / \mathrm{grp})$ were inoculated intravenously with $2 \times 10^{4}$ CFU F. tularensis. After $24 \mathrm{~h}$, infected mice were euthanized along with uninfected controls ( $n=3 / g r p)$. (A) Mean proportion of splenic monocytes (CD11b+, Ly6C ${ }^{\text {hi }}$ population) \pm SD was determined as in Figure $1 \mathrm{~A}$ in control and F. tularensis-infected WT BM $\rightarrow$ WT and IFN $\gamma \mathrm{R}-/-\mathrm{BM} \rightarrow \mathrm{WT}$ mice. (B) Mean protein levels $\pm \mathrm{SD}$ of MCP1 from spleen homogenates and sera of WT and IFN $\gamma$ R-deficient mice. (C) F. tularensis CFU recovered from spleens of WT $\mathrm{BM} \rightarrow \mathrm{WT}$ and IFN $\gamma \mathrm{R}-/-\mathrm{BM} \rightarrow \mathrm{WT}$ mice $(n=3 / \mathrm{grp})$ three days after inoculation with $2 \times 10^{4}$ CFU F. tularensis. ${ }^{*} p<0.05$ WT $\mathrm{BM} \rightarrow \mathrm{WT}$ versus IFN $\gamma \mathrm{R}-/-\mathrm{BM} \rightarrow \mathrm{WT}$ mice, ${ }^{\ddagger} p<0.01 \mathrm{WT} \mathrm{BM} \rightarrow \mathrm{WT}$ versus IFN $\gamma \mathrm{R}-1-\mathrm{BM} \rightarrow \mathrm{WT}$ mice. Data are representative of at least three repeated experiments. 
TLR2 ligand (Figure 3D). Taken together, these data suggest that IFN $\gamma$ is sufficient to activate $\mathrm{MCP}-1$ production in BMMs. Furthermore, the IFN $\gamma$-dependent activation of MCP-1 can be amplified by TLR2 or F. tularensis stimulation.

MyD88-dependent signaling is required for IFNy-driven $M C P-1$ production and expansion of splenic monocyte populations in response to systemic F. tularensis challenge

Previously published work has demonstrated that the adaptor protein MyD88, which is utilized by IL-1R, IL$18 \mathrm{R}$, and most TLRs to initiate signaling, is a critical mediator of host defense against $F$. tularensis $[29,30]$. Consistent with these findings, we observed that MyD88deficient mice are highly susceptible to systemic $F$. tularensis challenge and cannot effectively clear bacteria
(Supplementary information, Figure S2). However, we specifically wanted to ascertain whether MyD88 was required for the IFN $\gamma \mathrm{R}$-dependent MCP-1 production and expansion of splenic monocytes. After systemic F. tularensis challenge, MyD88-deficient mice had the same defect in expansion of splenic monocyte populations (Figure $4 \mathrm{~A}$ ) as we previously observed in IFN $\gamma \mathrm{R}$-deficient mice (Figure 1A and $1 \mathrm{C}$ ). Furthermore, we found that MCP-1 protein levels in the spleens of infected MyD88-deficient mice were substantially decreased compared with WT mice (Figure 4B). Given these findings, we hypothesized that MyD88 may either be required for production of IFN $\gamma$ or that it may be necessary for production of MCP1 via a different mechanism that does not impact IFN $\gamma$ production. Therefore, IFN $\gamma$ levels in the sera of WT and MyD88-deficient mice were measured after systemic challenge with $F$. tularensis. Consistent with prior
A

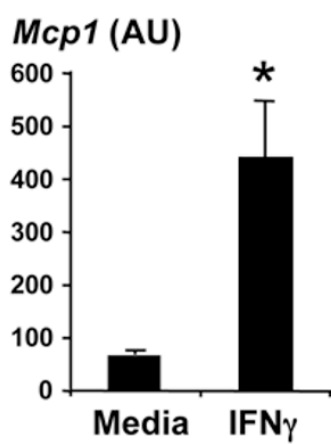

C

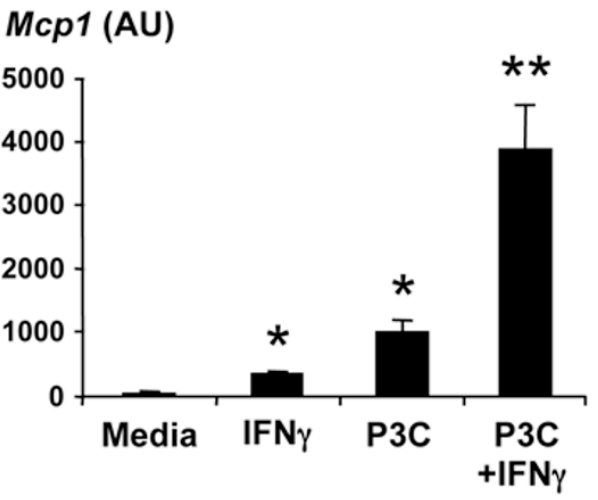

B

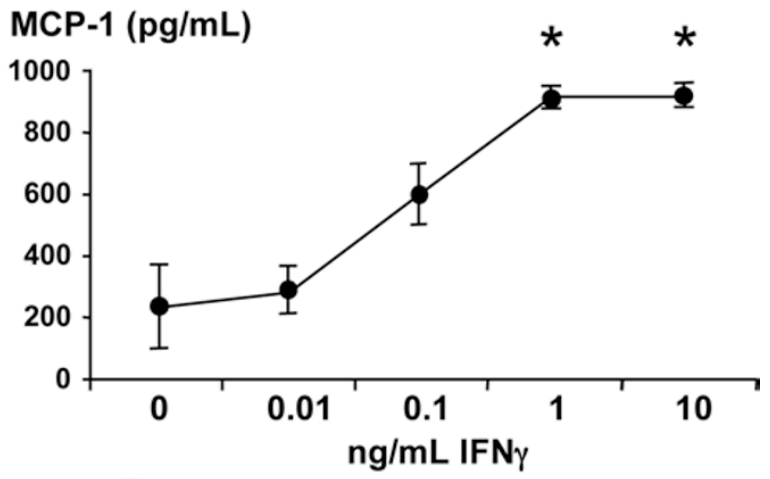

MCP-1 $(\mathrm{pg} / \mathrm{mL})$

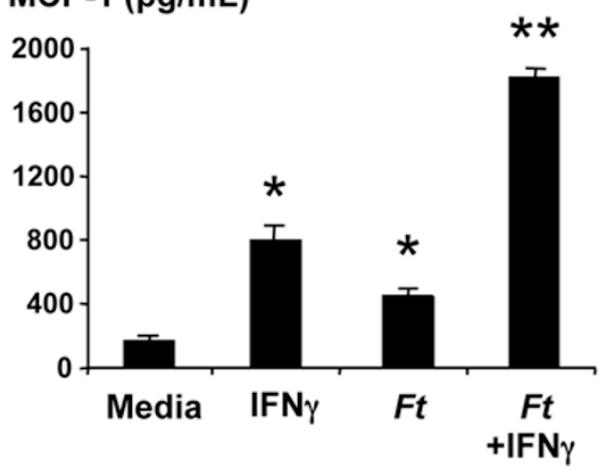

Figure 3 IFN $\gamma$ R signaling induces MCP-1 production in bone marrow-derived cells and acts synergistically with innate sensing mechanisms to induce increased levels of MCP-1 in vitro. (A) Mean MCP-1 mRNA levels \pm SD from WT control BMMs and WT BMMs stimulated with $0.1 \mathrm{ng} / \mathrm{ml} \mathrm{IFN} \gamma$ for $6 \mathrm{~h}$ prior to harvest. (B) Mean MCP-1 protein levels \pm SD from supernatants of WT control BMMs and WT BMMs stimulated with the indicated concentrations of IFN $\gamma$ for $24 \mathrm{~h}$ prior to harvest. (C) Mean MCP-1 mRNA levels from control BMMs and BMMs stimulated with $1 \mathrm{ng} / \mathrm{ml} \mathrm{IFN} \gamma, 0.01 \mathrm{ng} / \mathrm{ml}$ Pam3CysK, or both for $6 \mathrm{~h}$ prior to harvest. (D) Mean MCP-1 protein levels from supernatants of control BMMs and BMMs stimulated with $1 \mathrm{ng} / \mathrm{ml}$ IFN $\gamma$, MOI $1 F$. tularensis, or both for $24 \mathrm{~h}$ prior to harvest. ${ }^{*} p<0.05$ versus media control, ${ }^{* *} p<0.05$ versus media control and other data points. Data are representative of at least three repeated experiments. 
published results [31], there was no detectable IFN $\gamma$ in the sera of infected MyD88-deficient mice compared to robust IFN $\gamma$ production in WT mice (Figure 4C). These findings suggest that the production of IFN $\gamma$ is dependent upon MyD88-mediated signals.

CCR2-deficient mice exhibit defective expansion of splenic monocyte populations and host defense in response to F. tularensis challenge

To directly test whether IFN $\gamma$ R-dependent MCP-1 production plays an important role in the mobilization of monocytes to the spleen in the context of systemic $F$. tularensis challenge in vivo, we examined the expansion of CD11b+ Ly6 $\mathrm{C}^{\text {hi }}$ monocyte populations in the spleens of CCR2-deficient mice. These mice lack the receptor for MCP-1 and are thus unable to mobilize monocytes to the periphery upon bacterial challenge [7, 31, 32]. Similar to IFN $\gamma R$-deficient mice, we observed defective expansion of splenic monocytes in CCR2-deficient mice after $F$. tularensis challenge (Figure 5A). These findings directly link MCP-1 to the IFN $\gamma$-dependent splenic monocyte expansion observed in vivo. We then hypothesized that if the monocyte populations mobilized to the spleen are critical to host defense against $F$. tularensis, then infected CCR2-deficient mice should have increased bacterial CFU in their spleens. We therefore challenged WT, IFN $\gamma$ R-deficient, and CCR2-deficient mice with $F$. tularensis, and subsequently assessed bacterial CFU in spleens from these mice. Notably, there were approximately tenfold more $F$. tularensis CFU in the spleens of
CCR2-deficient mice compared with WT control mice three days after inoculation, indicating that CCR2-driven mobilization of monocytes to the spleen is required for control of bacterial numbers (Figure 5B). Interestingly, we observed tenfold more bacterial CFU in the spleens of IFN $\gamma \mathrm{R}$-deficient mice than in CCR2-deficient mice, suggesting that IFN $\gamma \mathrm{R}$ is required for additional host defense functions aside from driving the CCR2-dependent expansion of splenic monocytes (Figure 5B). Taken together, these observations define a host defense circuit in which IFN $\gamma \mathrm{R}$ signaling in BM-derived cells activates production of MCP-1, which is in turn required for the mobilization of monocytes to the spleen and effective control of $F$. tularensis numbers there.

IFN $\gamma$ R-deficient and CCR2-deficient mice have severe defects in bacterial clearance and in production of TNF- $\alpha$ and nitric oxide following systemic F. tularensis challenge

Prior work has suggested that upon reaching areas of infection, monocytes produce TNF- $\alpha$ and NO, critical effector mechanisms for clearing intracellular bacterial pathogens such as F. tularensis [10]. Thus, we examined whether CCR2-dependent mobilization of monocytes to the spleen was required for TNF- $\alpha$ and NO production in response to $F$. tularensis. Thus, we harvested spleens from WT, IFN $\gamma \mathrm{R}$-deficient and CCR2-deficient mice $24 \mathrm{~h}$ after $F$. tularensis challenge to assess production of TNF- $\alpha$ and NO. We found that intracellular TNF- $\alpha$ production in $\mathrm{CD} 11 \mathrm{~b}+$ splenocytes harvested from $F$. tularensis-infected IFN $\gamma \mathrm{R}$-deficient and CCR2-deficient
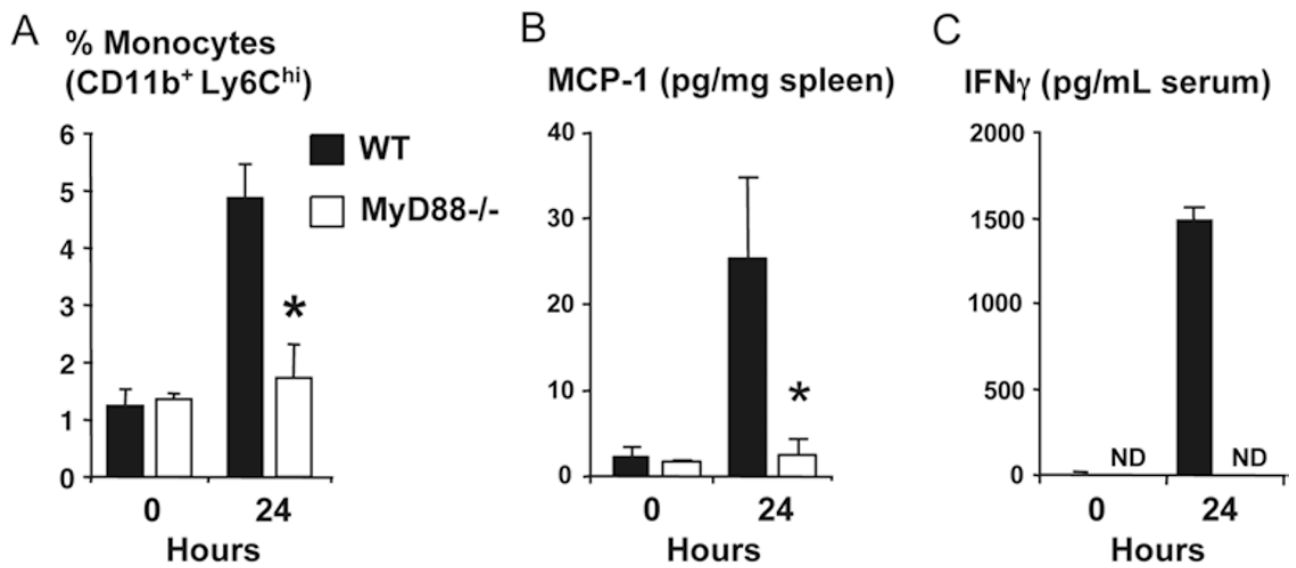

Figure 4 IFN $\gamma$-driven MCP-1 expression and expansion of splenic monocyte populations in response to systemic $F$. tularensis challenge occurs via MyD88-dependent signaling. WT and MyD88-/- mice ( $n=3 / \mathrm{grp})$ were inoculated intravenously with 2 $\times 10^{4}$ CFU F. tularensis. After $24 \mathrm{~h}$, infected mice were euthanized along with uninfected $(0 \mathrm{~h})$ controls $(n=3 / \mathrm{grp})$. (A) Mean proportion of splenic monocytes $\left(\mathrm{CD} 11 \mathrm{~b}+\right.$, Ly6 $\mathrm{C}^{\text {hi }}$ population) $\pm \mathrm{SD}$ was determined as in Figure $1 \mathrm{~A}$ in control and $\mathrm{F}$. tularensis-infected WT and MyD88-/- mice. (C) Mean mRNA levels \pm SD of MCP-1 from control and infected WT and MyD88-/mice. (D) Mean protein levels \pm SD of IFN $\gamma$ in sera from control and infected WT and MyD88-/- mice. ND, not detected. ${ }^{*} p<$ 0.05 WT versus MyD88-/- mice (Student's $t$-test). Data are representative of at least three repeated experiments. 
A

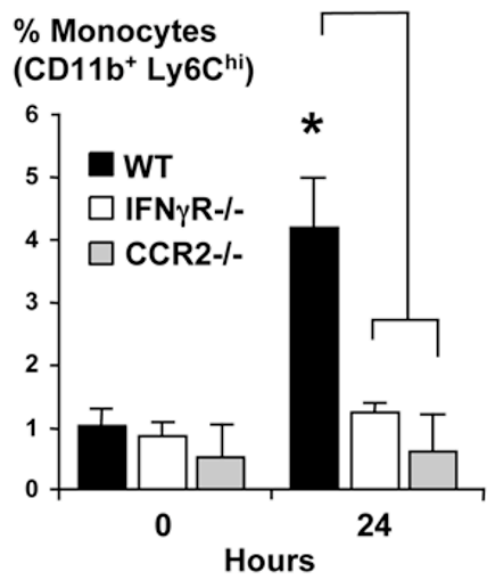

B

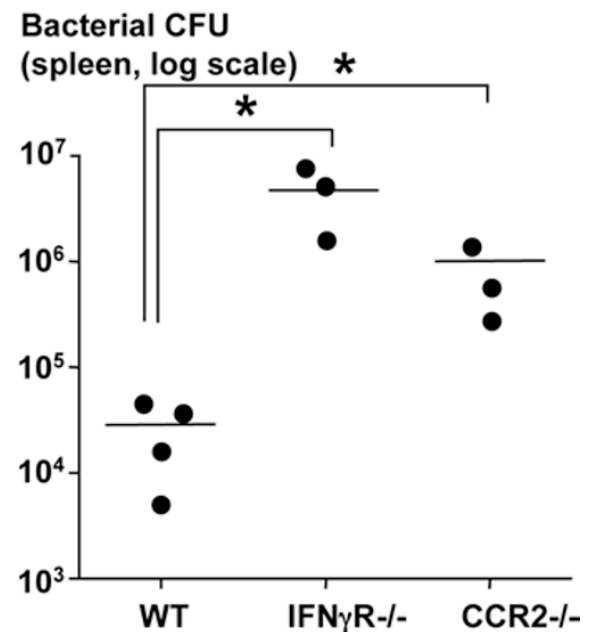

Figure 5 CCR2-deficient mice exhibit defective expansion of splenic monocyte populations and host defense in response to systemic F. tularensis challenge. WT mice, IFN $\gamma \mathrm{R}-1-$ mice, and CCR2-l- mice $(n=3 / \mathrm{grp})$ were inoculated intravenously with $2 \times 10^{4} \mathrm{CFU}$ F. tularensis. After $24 \mathrm{~h}$, infected mice were euthanized along with uninfected controls $(n=3 / \mathrm{grp})$. (A) Mean proportion of splenic monocytes (CD11b+, Ly6 $C^{\text {hi }}$ population) \pm SD was determined as in Figure $1 A$ in control and $F$. tularensis-infected WT mice, IFN $\gamma \mathrm{R}-/-$ mice, and CCR2-I- mice. (B) F. tularensis CFU recovered from the spleens of WT, IFN $\gamma \mathrm{R}-/-$ mice, and CCR2-I- mice three days after intravenous inoculation with $2 \times 10^{4}$ CFU F. tularensis. * $p<0.05$ WT versus IFN $\gamma R-/-$ or CCR2-I- mice. Data are representative of at least three repeated experiments.

mice was severely impaired compared with the TNF- $\alpha$ production in WT control mice (Figure 6A). Furthermore, to determine whether these splenocytes were capable of producing NO, cells from $F$. tularensis-challenged WT, IFN $\gamma$ R-deficient, and CCR2-deficient mice were cultured ex vivo with heat-killed $F$. tularensis (HKFT), and culture supernatants were evaluated for production of nitrite, a NO breakdown product. Similar to TNF- $\alpha$ levels, there were significantly lower levels of nitrite in the supernatants of HKFT-stimulated splenocytes from IFN $\gamma$ R-deficient and CCR2-deficient mice relative to WT mice (Figure 6B). Taken together, these data suggest that IFN $\gamma$ R-dependent CCR2 signaling is required for the mobilization of monocytes to the spleen, where they act as critical producers of TNF- $\alpha$ and NO and mediate clearance of $F$. tularensis infection.

\section{Discussion}

In the present study, we describe a host defense circuit activated by $F$. tularensis whereby MyD88 mediates production of IFN $\gamma$, which in turn signals via its receptor in BM-derived cells, resulting in the production of MCP-1 and subsequent CCR2-dependent mobilization of monocyte cells to the spleen. There, mobilized monocytes subsequently produce TNF- $\alpha$ and $\mathrm{NO}$ and mediate clearance of $F$. tularensis.
The role of IFN $\gamma \mathrm{R}$ signaling in the activation of innate immune responses is critical to host defense in a wide range of in vivo models of intracellular bacterial infection, including systemic Mycobacterium tuberculosis, and L. monocytogenes infections [6, 33, 34]. Likewise, IFN $\gamma \mathrm{R}$ signaling is absolutely required for control of $F$. tularensis infection, and mice lacking IFN $\gamma$ are rendered moribund 5-7 days after challenge, consistent with our observations in this study [19, 35]. IFN $\gamma$ activates a wide range of antimicrobial mechanisms, including induction of iNOS and subsequent NO production, which is critical for the destruction of pathogenic organisms [36]. However, the question of how IFN $\gamma$ may fit into the wider signaling network that regulates the trafficking of immune cells, particularly myeloid cells, during the innate immune response to infection has not been well characterized. Thus, in the present study, we have uncovered a novel function of IFN $\gamma \mathrm{R}$ signaling as a critical regulator of monocyte trafficking in response to systemic F. tularensis infection.

Our data show that IFN $\gamma$ R-deficient mice have decreased systemic levels of MCP-1, which suggests that induction of MCP-1 is a mechanism by which IFN $\gamma$ orchestrates the mobilization of monocytes. We confirmed this mechanism by showing that mice deficient in CCR2, the MCP-1 receptor, also fail to mobilize monocytes to the spleen in response to F. tularensis challenge. Analy- 


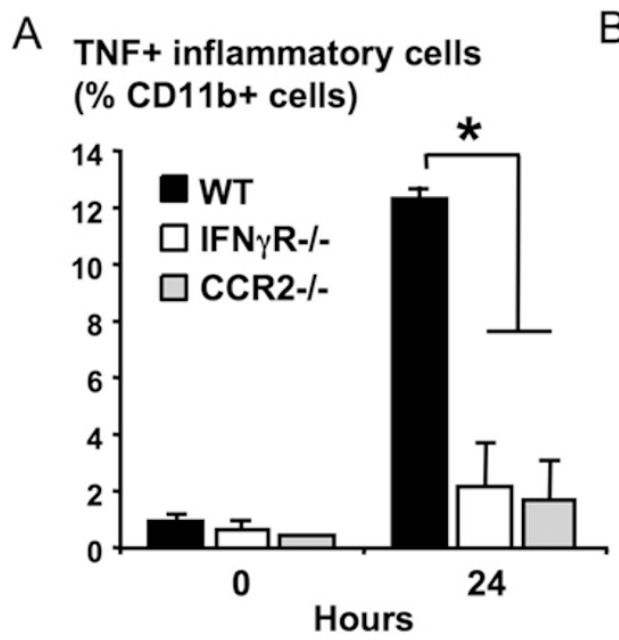

B

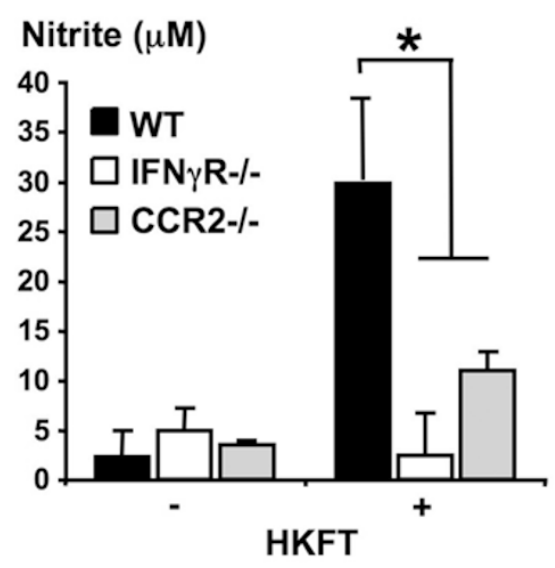

Figure 6 Mice deficient in CCR2 have similar defects as IFN $\gamma$ R-deficient mice in production of TNF- $\alpha$ and nitric oxide by inflammatory monocytes following systemic F. tularensis challenge. WT, IFN $\gamma \mathrm{R}-1-$ mice, and CCR2 $-/-$ mice $(n=3 / g r p)$ were inoculated intravenously with $2 \times 10^{4}$ CFU F. tularensis. (A) Mean proportion \pm SD of CD11b+ splenocytes producing TNF- $\alpha$ by intracellular staining from WT mice, IFN $\gamma \mathrm{R}-/-$ mice, and CCR2-/- mice. (B) Mean nitrite production by splenocytes harvested from WT mice, IFN $\gamma \mathrm{R}-/-$ mice, and CCR2-/- mice inoculated for $24 \mathrm{~h}$ with $\mathrm{F}$. tularensis and restimulated with heatkilled F. tularensis in culture. ${ }^{*} p<0.05$ WT versus IFN $\gamma R-/-$ or CCR2-/- mice. Data are representative of at least two repeated experiments.

sis of the MCP-1 promoter and enhancer has uncovered the presence of NF- $\mathrm{kB}$ and STAT-1 binding sites. In vitro studies have shown that MCP-1 is induced by a number of NF- $\mathrm{BB}$-activating inflammatory signals, including TNF- $\alpha$ and TLR signaling in a wide range of cell types, including myeloid cells [26, 37]. F. tularensis, like many other bacteria, contains a complex set of molecular motifs that can induce NF- $\kappa B$ activation via TLRs $[16,38$, 39]. We were therefore surprised that signaling via TLRs and other inflammatory cytokines activated by $F$. tularensis infection was not itself sufficient to drive MCP1 production in IFN $\gamma \mathrm{R}$-deficient mice. However, our in vitro experiments suggest that IFN $\gamma$ synergistically enhances otherwise modest $F$. tularensis-induced MCP1 production, suggesting that $F$. tularensis alone may not efficiently trigger an immune response, and thus IFN $\gamma$ functions as an amplifying loop, resulting in increased MCP-1 production and efficient mobilization of monocytes. Interestingly, $F$. tularensis LPS has been shown to be a weak activator of TLR signaling in vitro and in vivo [40-43]. Furthermore, F. tularensis has been shown to impair the production of inflammatory cytokines in infected cells [44]. Thus, IFN $\gamma \mathrm{R}$ signaling may serve to counteract these unique properties of $F$. tularensis, which may serve as possible immune evasion mechanisms, allowing the activation of a robust host defense response to F. tularensis. Further studies should directly compare how efficiently different intracellular bacteria, including
F. tularensis, or their components activate immune responses in monocytes and macrophages.

Our data demonstrate a role for MyD88-dependent signaling in the induction of IFN $\gamma$ and subsequent MCP1 production. These findings are consistent with previous work showing defective IFN $\gamma$ production in $F$. tularensis-infected MyD88-deficient mice [29]. MyD88 functions downstream of three major host defense signaling mechanisms, TLRs, IL-1R, and IL-18R [30]. Prior studies have suggested a role for TLR2 in the recognition of $F$. tularensis lipoproteins, and while we have not observed decreased IFN $\gamma$ production in TLR2-deficient mice (our unpublished observation), it is unclear whether TLR2 is the only TLR to recognize F. tularensis [16, 45]. Interestingly, F. tularensis-infected IL-18R-deficient mice produce less IFN $\gamma$ (our unpublished observation), suggesting that signaling downstream of IL-18R may be part of the MyD88-dependent host defense mechanism described in our study. IL-18, in conjunction with IL12 , activates IFN $\gamma$ production by NK cells and $\mathrm{T}$ lymphocytes [46]. Both NK and T cell populations produce IFN $\gamma$ in response to $F$. tularensis, suggesting that NK cells and $\mathrm{T}$ cells may be critical sources of IFN $\gamma$ during the host response against $F$. tularensis infection [23, 35, 47]. Interestingly, work using Rag1-deficient mice suggests that early IFN $\gamma$ production can occur independently of $\mathrm{T}$ lymphocytes, as the proportion of IFN $\gamma$-producing cells is not decreased in these mice [48]. Conversely, 
mice depleted of NK cells have lower proportions of IFN $\gamma$-producing cells three days after $F$. tularensis inoculation $[23,47]$. Notably, one study demonstrated that the efficient formation of myeloid granulomas in the liver after $F$. tularensis infection required both IFN $\gamma$ and NK cells, linking NK cell-mediated IFN $\gamma$ production to myeloid cell trafficking [23]. While these findings suggest that NK cells in particular may be largely responsible for early IFN $\gamma$ production, depletion of NK cells in mice prior to infection did not increase their susceptibility to $F$. tularensis challenge, suggesting that other cell types may produce enough IFN $\gamma$ to sufficiently activate host defense $[47,49]$. Interestingly, IFN $\gamma$ expression in response to $F$. tularensis infection has been detected in dendritic cells and neutrophils, as well as hybrid NKDC populations, suggesting that IFN $\gamma$ production by these non-lymphoid cell types may contribute to early activation of host defense against $F$. tularensis in the absence of T or NK cells [48]. Based on these studies, we speculate that MyD88dependent IL-18R signaling, predominantly in NK and $\mathrm{T}$ cells, is a likely mechanism by which IFN $\gamma$ production is activated in response to F. tularensis. Furthermore, the observation that myeloid populations can produce IFN $\gamma$ in response to $F$. tularensis raises the interesting possibility that IL-18R/MyD88-dependent IFN $\gamma$ production by these cells could contribute to the induction of MCP-1 expression.

We found that CCR2-deficient mice failed to mobilize monocytes to the spleen after $F$. tularensis challenge, and have significantly elevated bacterial numbers in the spleen compared to WT mice. These observations serve as direct evidence that monocytes are critical for efficient control of $F$. tularensis infection. CCR2-deficient mice serve as a compelling and widely used model for studying the specific contribution of monocytes to host defense, as these mice fail to mobilize monocytes to the periphery, including the spleen, upon infection or inflammation [7, 31, 32]. Importantly, the number and function of other cell types, including lymphocytes, neutrophils, tissue macrophages, and monocytes themselves, are not greatly perturbed by loss of CCR2 signaling at the steady state, making use of this model particularly advantageous [7, 31, 32]. The defects in host defense we have observed using CCR2-deficient mice to study the role of monocytes in response to $F$. tularensis infection are consistent with published studies using CCR2-deficient mice, as well as mice deficient in MCP-1 and other individual CCR2 ligands, to probe the role of monocytes in response to L. monocytogenes infection $[5,7,50]$. Collectively, these findings suggest that monocytes are critical mediators of the innate immune response against intracellular bacterial pathogens in general.
Monocytes are thought to control infection by intracellular bacteria in part via production of TNF- $\alpha$, which activates immune responses via autocrine and paracrine mechanisms; they also activate direct antimicrobial effector mechanisms such as production of NO $[5,10]$. Both mechanisms have been shown to be critical for clearance of $F$. tularensis [51, 52]. We have shown that production of TNF- $\alpha$ and NO is defective in IFN $\gamma \mathrm{R}-$ and CCR2-deficient mice, which at least in part explains the elevated $F$. tularensis CFU we found in the spleens of these mice. Notably, we found that IFN $\gamma$ R-deficient mice had nearly a $\log$ more $F$. tularensis in their spleens than CCR2-deficient mice, suggesting that IFN $\gamma$ activates a wider set of antimicrobial mechanisms, in which monocytes play a critical part.

Collectively, our findings describe a novel role for MyD88-dependent IFN $\gamma \mathrm{R}$ signaling in the activation of $\mathrm{MCP}-1$ production, leading to mobilization and activation of monocytes, which in turn mediate efficient host defense in part via TNF- $\alpha$ and NO production. Notably, recent work has uncovered a possible role for IFN $\gamma$ in the activation of hematopoietic stem and progenitor cell proliferation in vivo and in vitro [53]. These findings suggest that IFN $\gamma$ may trigger the de novo generation of myeloid cells to replenish the immune system in addition to inducing MCP-1-dependent mobilization of extant myeloid populations from the BM to the periphery. Furthermore, CCR2 has been shown to regulate the migration of hematopoietic progenitor cells from the BM to the periphery in response to inflammation [54]. Combining these findings with our results, the IFN $\gamma \mathrm{R} / \mathrm{CCR} 2$ signaling axis is a critical mechanism for the activation and remodeling of both immature and mature BM and peripheral hematopoietic compartments in response to inflammation and intracellular pathogen infection.

\section{Materials and Methods}

\section{F. tularensis preparation}

The F. tularensis live vaccine strain (LVS) was generously provided by Dr Karen Elkins. F. tularensis from frozen aliquots was streaked onto rabbit cysteine blood agar plates (Remel, Lenexa, $\mathrm{KS}$ ) and individual colonies of $F$. tularensis were grown overnight in Mueller-Hinton Broth (MHB) supplemented with 1\% Isovitalex (BD Biosciences), 0.1\% glucose and ferric pyrophosphate (Sigma-Aldrich). Bacteria were harvested while in mid-log phase as determined by optical density at $600 \mathrm{~nm}$ wavelength in a spectrophotometer (Beckman-Coulter), washed twice with sterile PBS and diluted with sterile PBS for infection. Bacterial CFUs were verified by plating dilutions onto rabbit cysteine blood agar.

\section{Mice}

All procedures described herein were approved by and conducted in compliance with the UCLA Animal Research Committee. 
6-8 week-old female C57BL/6J (WT), IFN $\gamma$ R-deficient, IFN $\alpha \beta R$ deficient, and CCR2-deficient mice were obtained from the Jackson Laboratories. MyD88-deficient mice and control littermates were obtained from Dr Shizuo Akira. Mice were housed and bred in the UCLA vivarium in specific pathogen-free conditions.

\section{Intravenous F. tularensis systemic infection model}

For in vivo infections, mice were inoculated via the lateral tail vein with $2 \times 10^{4} \mathrm{CFU} \mathrm{F}$. tularensis grown to log-phase and prepared as described above in a $200-\mu 1$ bolus using a 28 -gauge syringe (Becton-Dickenson), and euthanized $24 \mathrm{~h}$ after challenge. Groups of three mice were used in each experiment. For quantification of $F$. tularensis CFUs, spleens were homogenized to singlecell suspension and serially plated on rabbit cysteine blood agar plates for colony counting.

\section{Generation of BM chimeras}

BM reconstitution experiments were performed as previously described [55]. Whole BM was flushed from the femurs and tibiae of donor mice and depleted of RBCs using ACK lysis buffer $(0.15$ $\mathrm{M}$ ammonium chloride $\left(\mathrm{NH}_{4} \mathrm{Cl}\right), 1 \mathrm{mM}$ potassium bicarbonate $\left(\mathrm{KHCO}_{3}\right)$, and $0.1 \mathrm{mM}$ EDTA $\left.(\mathrm{pH} 7.3)\right)$. BM cells were subsequently washed twice in PBS and counted on a hemocytometer. WT recipient mice were lethally irradiated with $1100 \mathrm{Rad}$ and reconstituted $24 \mathrm{~h}$ later by injection of $1 \times 10^{7} \mathrm{WT}$ or IFN $\gamma \mathrm{R}$ deficient donor BM cells via the lateral tail vein. Mice were maintained in autoclaved cages, given irradiated feed and were maintained on sulfamethoxazole and trimethoprim oral suspension (TMS; $48 \mathrm{mg} / \mathrm{ml}$ in drinking water) for three weeks after irradiation. Mice were used for experimentation 8 weeks after BM reconstitution.

\section{Flow cytometry}

Spleens were homogenized into a single-cell suspension and depleted of RBC using ACK lysis buffer. Splenocytes were resuspended in staining media consisting of PBS containing $2 \%$ FBS and stained with anti-CD11b-APC or FITC, and anti-Ly6CFITC or anti-Ly6C-biotin on ice for $30 \mathrm{~min}$. All antibodies and SA-APC fluorochrome were obtained from BD Pharmingen (San Jose, CA). Cells were then washed and fixed with $2 \%$ PFA prior to analysis on a FACScalibur flow cytometer. For intracellular stains, $2 \times 10^{5}$ splenocytes were resuspended in DMEM containing $10 \%$ FBS containing 1:1 000 BD GolgiPlug and $2 \times 10^{6}$ heat-killed F. tularensis for $4 \mathrm{~h}$ in a $37^{\circ} \mathrm{C}$ humidified incubator. Cells were subsequently stained for CD11b, fixed and permeabilized using BD Cytofix/Cytoperm, and stained for intracellular TNF- $\alpha$ using an anti-TNF- $\alpha$-PE antibody for $30 \mathrm{~min}$ on ice. Cells were washed with BD PermWash and analyzed on a BD FACSCalibur. All flow cytometry data were analyzed using FlowJo (TreeStar, Inc., Palo Alto, CA, USA).

\section{In vitro $F$. tularensis infection}

WT BMMs were generated from whole mouse BM as previously described [56]. For in vitro infections, bacteria were diluted in DMEM and added at an MOI of 1 to $1 \times 10^{6} \mathrm{BM}$ macrophages (for Q-PCR analysis) or $2 \times 10^{4} \mathrm{BM}$ macrophages (for ELISA analysis) cultured in antibiotic-free media containing 5\% heatinactivated FBS. Bacteria were spun onto cells at $2000 \mathrm{rpm}$ for $10 \mathrm{~min}$ in a Beckman centrifuge at room temperature. Media was washed off $1 \mathrm{~h}$ later and replaced with media containing $2 \mu \mathrm{g} /$ $\mathrm{ml}$ gentamicin (Sigma-Aldrich). Pam3Cys (Sigma-Aldrich) was added at $0.01 \mathrm{ng} / \mathrm{ml}$; recombinant murine IFN $\gamma$ (R\&D Systems) was added at concentrations ranging from 0.01 to $10 \mathrm{ng} / \mathrm{ml}$. For MCP1 mRNA expression analysis experiments, cells were washed with PBS $6 \mathrm{~h}$ later and harvested in $1 \mathrm{ml}$ of Trizol (Invitrogen). For MCP-1 ELISA, supernatants were recovered $24 \mathrm{~h}$ later.

\section{Cytokine expression analysis by quantitative RT-PCR}

Total RNA was harvested from BMMs or washed splenocytes using Trizol (Invitrogen) according to the manufacturer's instructions. cDNA was generated from RNA using iScript (Bio-Rad). Quantitative RT-PCR analysis of MCP-1 mRNA levels was carried out on an iCycler (Bio-Rad) using Sybr Green $2 \times$ Master Mix (Applied Biosystems). Primer sequences used are MCP1 Fwd 5'-GCTGACCCCAAGAAGGAATG-3', Rev 5'-GAAGACCTTAGGGCAGATGCA-3'. Data were normalized to L32 mRNA levels.

\section{ELISAS}

ELISA development kits for MCP-1 (eBioscience, San Diego, CA, USA) were used according to the manufacturer's instructions and analyzed using a 96-well plate spectrophotometer (ThermoFisher, Waltham, MA, USA). Spleens to be analyzed for cytokine levels by ELISA were weighed and homogenized in $0.5 \mathrm{ml}$ PBS containing $0.05 \%$ Triton X-100 and centrifuged to remove debris. Supernatants were collected from 96-well plates and centrifuged to remove debris. All samples were plated in duplicate. Cytokine concentrations obtained from spleen homogenates were normalized to spleen mass.

\section{Statistical analysis}

The unpaired Student's $t$-test was used to compare groups; $P$-values of 0.05 or less were considered to be statistically significant.

\section{Acknowledgments}

We would like to thank Dr Karen Elkins (US Food and Drug Administration (FDA), USA) for kindly providing the F.tularensis LVS. We also thank Dr Shizuo Akira (Osaka University, Japan) for the generous gift of MyD88-deficient mice. This work was supported by grants R01 AI056154, R01 CA87924, and R01 AI052359 (to GC). This work was also supported by the Ruth L Kirschstein Research Service Award GM 007185 (to EMP).

\section{References}

1 Fearon DT, Locksley RM. The instructive role of innate immunity in the acquired immune response. Science 1996; 272:50-53.

2 Takeda K, Akira S. Toll-like receptors in innate immunity. Int Immunol 2005; 17:1-14.

3 Pedra JH, Cassel SL, Sutterwala FS. Sensing pathogens and danger signals by the inflammasome. Curr Opin Immunol 2009; 21:10-16

4 Fritz JH, Girardin SE. How Toll-like receptors and Nod-like receptors contribute to innate immunity in mammals. $J$ Endotoxin Res 2005; 11:390-394. 
5 Serbina NV, Jia T, Hohl TM, Pamer EG. Monocyte-mediated defense against microbial pathogens. Annu Rev Immunol 2008; 26:421-452.

6 Busch DH, Vijh S, Pamer EG. Animal model for infection with Listeria monocytogenes. Curr Protoc Immunol 2001; Chapter 19:Unit 19.9.

7 Serbina NV, Pamer EG. Monocyte emigration from bone marrow during bacterial infection requires signals mediated by chemokine receptor CCR2. Nat Immunol 2006; 7:311-317.

8 Huffnagle GB, Traynor TR, McDonald RA, et al. Leukocyte recruitment during pulmonary Cryptococcus neoformans infection. Immunopharmacology 2000; 48:231-236.

9 Amano H, Morimoto K, Senba M, et al. Essential contribution of monocyte chemoattractant protein-1/C-C chemokine ligand-2 to resolution and repair processes in acute bacterial pneumonia. J Immunol 2004; 172:398-409.

10 Serbina NV, Salazar-Mather TP, Biron CA, Kuziel WA, Pamer EG. TNF/iNOS-producing dendritic cells mediate innate immune defense against bacterial infection. Immunity 2003; 19:59-70.

11 Boehm U, Klamp T, Groot M, Howard JC. Cellular responses to interferon-gamma. Annu Rev Immunol 1997; 15:749-795.

12 Leite-de-Moraes MC, Dy M. Natural killer T cells: a potent cytokine-producing cell population. Eur Cytokine Netw 1997; 8:229-237.

13 Schoenborn JR, Wilson CB. Regulation of interferon-gamma during innate and adaptive immune responses. Adv Immunol 2007; 96:41-101.

14 Kleinert H, Schwarz PM, Forstermann U. Regulation of the expression of inducible nitric oxide synthase. Biol Chem 2003; 384:1343-1364.

15 Shtrichman R, Samuel CE. The role of gamma interferon in antimicrobial immunity. Curr Opin Microbiol 2001; 4:251259.

16 Cole LE, Shirey KA, Barry E, et al. Toll-like receptor 2-mediated signaling requirements for Francisella tularensis live vaccine strain infection of murine macrophages. Infect Immun 2007; 75:4127-4137.

17 Collazo CM, Sher A, Meierovics AI, Elkins KL. Myeloid differentiation factor-88 (MyD88) is essential for control of primary in vivo Francisella tularensis LVS infection, but not for control of intramacrophage bacterial replication. Microbes Infect 2006; 8:779-790.

18 Sjostedt A, Conlan JW, North RJ. Neutrophils are critical for host defense against primary infection with the facultative intracellular bacterium Francisella tularensis in mice and participate in defense against reinfection. Infect Immun 1994; 62:2779-2783.

19 Anthony LS, Ghadirian E, Nestel FP, Kongshavn PA. The requirement for gamma interferon in resistance of mice to experimental tularemia. Microb Pathog 1989; 7:421-428.

20 Anthony LS, Morrissey PJ, Nano FE. Growth inhibition of Francisella tularensis live vaccine strain by IFN-gammaactivated macrophages is mediated by reactive nitrogen intermediates derived from L-arginine metabolism. J Immunol 1992; 148:1829-1834.

21 Elkins KL, Colombini SM, Meierovics AI, Chu MC, Chou AY, Cowley SC. Survival of secondary lethal systemic Francisella LVS challenge depends largely on interferon gamma.
Microbes Infect 2010; 12:28-36.

22 Sjostedt A, North RJ, Conlan JW. The requirement of tumour necrosis factor-alpha and interferon-gamma for the expression of protective immunity to secondary murine tularaemia depends on the size of the challenge inoculum. Microbiology 1996; 142 (Pt 6):1369-1374.

23 Bokhari SM, Kim KJ, Pinson DM, Slusser J, Yeh HW, Parmely MJ. NK cells and gamma interferon coordinate the formation and function of hepatic granulomas in mice infected with the Francisella tularensis live vaccine strain. Infect Immun 2008; 76:1379-1389.

24 Billiau A, Matthys P. Interferon-gamma: a historical perspective. Cytokine Growth Factor Rev 2009; 20:97-113.

25 Fortier AH, Slayter MV, Ziemba R, Meltzer MS, Nacy CA. Live vaccine strain of Francisella tularensis: infection and immunity in mice. Infect Immun 1991; 59:2922-2928.

26 Ping D, Jones PL, Boss JM. TNF regulates the in vivo occupancy of both distal and proximal regulatory regions of the MCP-1/JE gene. Immunity 1996; 4:455-469.

27 Satriano JA, Hora K, Shan Z, Stanley ER, Mori T, Schlondorff $\mathrm{D}$. Regulation of monocyte chemoattractant protein-1 and macrophage colony-stimulating factor-1 by IFN-gamma, tumor necrosis factor-alpha, IgG aggregates, and cAMP in mouse mesangial cells. J Immunol 1993; 150:1971-1978.

28 Martin CA, Dorf ME. Differential regulation of interleukin-6, macrophage inflammatory protein-1, and JE/MCP-1 cytokine expression in macrophage cell lines. Cell Immunol 1991; 135:245-258.

29 Collazo CM, Sher A, Meierovics AI, Elkins KL. Myeloid differentiation factor-88 (MyD88) is essential for control of primary in vivo Francisella tularensis LVS infection, but not for control of intra-macrophage bacterial replication. Microbes Infect 2006; 8:779-790.

30 Medzhitov R, Preston-Hurlburt P, Kopp E, et al. MyD88 is an adaptor protein in the hToll/IL-1 receptor family signaling pathways. Mol Cell 1998; 2:253-258.

31 Kurihara T, Warr G, Loy J, Bravo R. Defects in macrophage recruitment and host defense in mice lacking the CCR2 chemokine receptor. J Exp Med 1997; 186:1757-1762.

32 Boring L, Gosling J, Chensue SW, et al. Impaired monocyte migration and reduced type 1 (Th1) cytokine responses in C-C chemokine receptor 2 knockout mice. J Clin Invest 1997; 100:2552-2561.

33 Flynn JL, Chan J, Triebold KJ, Dalton DK, Stewart TA, Bloom BR. An essential role for interferon gamma in resistance to Mycobacterium tuberculosis infection. J Exp Med 1993; 178:2249-2254.

34 Serbina NV, Salazar-Mather TP, Biron CA, Kuziel WA, Pamer EG. TNF/iNOS-producing dendritic cells mediate innate immune defense against bacterial infection. Immunity 2003; 19:59-70.

35 Elkins KL, Rhinehart-Jones TR, Culkin SJ, Yee D, Winegar RK. Minimal requirements for murine resistance to infection with Francisella tularensis LVS. Infect Immun 1996; 64:3288-3293.

36 Karupiah G, Hunt NH, King NJ, Chaudhri G. NADPH oxidase, Nramp1 and nitric oxide synthase 2 in the host antimicrobial response. Rev Immunogenet 2000; 2:387-415.

37 Ping D, Boekhoudt GH, Rogers EM, Boss JM. Nuclear fac- 
tor-kappa B p65 mediates the assembly and activation of the TNF-responsive element of the murine monocyte chemoattractant-1 gene. J Immunol 1999; 162:727-734.

38 Kovarova H, Marcela A, Stulik J. Macrophage activating factors produced in the course of murine tularemia: effect on multiplication of microbes. Arch Immunol Ther Exp (Warsz) 1992; 40:183-190.

39 Cole LE, Santiago A, Barry E, et al. Macrophage proinflammatory response to Francisella tularensis live vaccine strain requires coordination of multiple signaling pathways. $J$ Immunol 2008; 180:6885-6891.

40 Vinogradov E, Perry MB, Conlan JW. Structural analysis of Francisella tularensis lipopolysaccharide. Eur J Biochem 2002; 269:6112-6118.

41 Dreisbach VC, Cowley S, Elkins KL. Purified lipopolysaccharide from Francisella tularensis live vaccine strain (LVS) induces protective immunity against LVS infection that requires B cells and gamma interferon. Infect Immun 2000; 68:19881996.

42 Duenas AI, Aceves M, Orduna A, et al. Francisella tularensis LPS induces the production of cytokines in human monocytes and signals via Toll-like receptor 4 with much lower potency than E. coli LPS. Int Immunol 2006; 18:785-795.

43 Hajjar AM, Harvey MD, Shaffer SA, et al. Lack of in vitro and in vivo recognition of Francisella tularensis subspecies lipopolysaccharide by Toll-like receptors. Infect Immun 2006; 74:6730-6738.

44 Telepnev M, Golovliov I, Grundstrom T, Tarnvik A, Sjostedt A. Francisella tularensis inhibits Toll-like receptor-mediated activation of intracellular signalling and secretion of TNF-alpha and IL-1 from murine macrophages. Cell Microbiol 2003; 5:41-51.

45 Malik M, Bakshi CS, Sahay B, Shah A, Lotz SA, Sellati TJ. Toll-like receptor 2 is required for control of pulmonary infection with Francisella tularensis. Infect Immun 2006; 74:36573662.

46 Dinarello CA. Interleukin-18. Methods 1999; 19:121-132.

47 Lopez MC, Duckett NS, Baron SD, Metzger DW. Early acti- vation of NK cells after lung infection with the intracellular bacterium, Francisella tularensis LVS. Cell Immunol 2004; 232:75-85.

48 De Pascalis R, Taylor BC, Elkins KL. Diverse myeloid and lymphoid cell subpopulations produce gamma interferon during early innate immune responses to Francisella tularensis live vaccine strain. Infect Immun 2008; 76:4311-4321.

49 Leiby DA, Fortier AH, Crawford RM, Schreiber RD, Nacy CA. In vivo modulation of the murine immune response to Francisella tularensis LVS by administration of anticytokine antibodies. Infect Immun 1992; 60:84-89.

50 Jia T, Serbina NV, Brandl K, et al. Additive roles for MCP-1 and MCP-3 in CCR2-mediated recruitment of inflammatory monocytes during Listeria monocytogenes infection. J Immunol 2008; 180:6846-6853.

51 Lindgren H, Stenman L, Tarnvik A, Sjostedt A. The contribution of reactive nitrogen and oxygen species to the killing of Francisella tularensis LVS by murine macrophages. Microbes Infect 2005; 7:467-475.

52 Chen W, KuoLee R, Shen H, Conlan JW. Susceptibility of immunodeficient mice to aerosol and systemic infection with virulent strains of Francisella tularensis. Microb Pathog 2004; 36:311-318.

53 Baldridge MT, King KY, Boles NC, Weksberg DC, Goodell MA. Quiescent haematopoietic stem cells are activated by IFN-gamma in response to chronic infection. Nature 2010; 465:793-797.

54 Si Y, Tsou CL, Croft K, Charo IF. CCR2 mediates hematopoietic stem and progenitor cell trafficking to sites of inflammation in mice. J Clin Invest 2010; 120:1192-1203.

55 Miller LS, Pietras EM, Uricchio LH, et al. Inflammasomemediated production of IL-1beta is required for neutrophil recruitment against Staphylococcus aureus in vivo. J Immunol 2007; 179:6933-6942.

56 Doyle SE, O'Connell RM, Miranda GA, et al. Toll-like receptors induce a phagocytic gene program through p38. J Exp Med 2004; 199:81-90.

(Supplementary information is linked to the online version of the paper on the Cell Research website.) 